KRZYSZTOF NYCZ

\title{
ÜBER DEN TEXT HINAUS: INTERTEXTUELLE BEZÜGE IN WIRTSCHAFTSTEXTEN
}

\begin{abstract}
A b s tra kt. Gegenstand dieses Beitrags ist das Konzept der Intertextualität, das von Julia Kristeva in den 1960er Jahren in die Literaturwissenschaft eingeführt wurde und in späterer Zeit für eine lebhafte Diskussion in der Literatur- und Sprachwissenschaft sorgte. Zuerst werden unterschiedliche linguistische Intertextualitätskonzepte dargestellt. Darauf folgt die Erklärung des Ansatzes der Textsortennetze. Zum Schluss werden intertextuelle Beziehungen zwischen ausgewählten Textsorten im Bereich der Börsenkommunikation dargestellt.
\end{abstract}

Schlüsselwörter: Intertextualität; Textsortennetze; Vernetzung von Fachtextsorten; Fachkommunikation; Börsenkommunikation.

\section{EINLEITUNG}

Das Phänomen der wechselseitigen Beziehungen und Abhängigkeiten, die zwischen Texten bestehen, wurde bereits in der Antike erkannt. Gleichwohl setzte der in den 1960er Jahren in die Literaturwissenschaft eingeführte Begriff der Intertextualität eine äußerst lebhafte Debatte in Gang. Traditionell werden darunter mehr oder weniger erkennbare und unterschiedlich explizit markierte Verweise auf andere Texte verstanden. Als mögliche Funktionen von solchen intertextuellen Bezugnahmen führt Janich („Intertextualität“ 177f.) Zusammenfassung, Nachahmung, Ergänzung, kritische Kommentierung, Verstärkung, argumentative Zuhilfenahme, Nachweis fremder Quellen, Markierung von Verbindlichkeit, Widerspruch, Persiflage und Parodierung sowie Aufmerksamkeitserregung auf. Allesamt dienen sie „der Erweiterung der Bedeutung des eigenen Textes“ (178), erfordern allerdings - wie Janich

Univ.-Prof. Dr. habil. KRzysztof NyCz - Universität Rzeszów, Institut für Neuphilologie; Korrespondenzadresse: Al. Rejtana 16c, 35-959 Rzeszów; e-mail: knycz@ur.edu.pl; ORCID: https://orcid.org/0000-0003-2976-1746. 
(178) treffend konstatiert - „eine besondere Rezeptionskompetenz [...], um Bezugnahmen nicht nur als solche erkennen, sondern auch den dadurch entstandenen Zuwachs an Textsinn entschlüsseln zu können.“

Im vorliegenden Aufsatz werden zunächst unterschiedliche Explikationen des Intertextualitätskonzeptes dargestellt. Darauf folgt die Erläuterung des Ansatzes der Vernetzung von (Fach-)Texten. Zum Schluss wird der Versuch unternommen, die Vielfalt intertextueller Bezüge am Beispiel ausgewählter Fachtextsorten aus dem Bereich der Börsenkommunikation darzustellen.

\section{LINGUISTISCHE INTERTEXTUALITÄTSKONZEPTE}

Das unbestrittene wissenschaftliche Verdienst, den Begriff der Intertextualität geprägt und in in den späten 1960er Jahren in die Literaturwissenschaft eingeführt zu haben, gebührt Julia Kristeva. ${ }^{1}$ Allerdings war Intertextualität lange Zeit kein Gegenstand linguistischer Forschung. Erst zu Beginn der 1980er Jahre führten R. de Beaugrande und W. Dressler ${ }^{2}$ bekanntlich das Konzept der Intertextualität aus den methodologischen Beschränkungen der Literaturwissenschaft heraus und öffneten es für linguistische Untersuchungen (vgl. Baumann, „Fachliche Intertextualität“ 125f.). ${ }^{3}$ Obwohl im Anschluss an R. de Beaugrande/W. Dressler eine Vielzahl von (text-)linguistisch orientieren Ansätzen ${ }^{4}$ entstanden ist, verweist U. Fix (451) darauf, dass in den 1990er Jahren Intertextualität als Stichwort in den Sachregistern von vielen bedeutenden textlinguistischen Arbeiten, etwa bei K. Brinker, vorerst nicht verzeichnet wird. Auch W. Heinemann/D. Viehweger behandeln Intertextualität eher am Rande, indem sie diese unter Rückgriff auf R. de Beaugrande/W. Dressler lediglich als eines der sieben Kriterien der Textualität nennen. Als nahezu bahnbrechend erweist sich in dieser Hinsicht die viel zitierte Monographie von S. Holthuis, in der sie eine „Taxonomie intertextueller Relationen“, eine konsistente und möglichst vollständige

\footnotetext{
${ }^{1}$ Auf eine vollständige Sichtung der gegenwärtig existierenden zahlreichen Intertextualitätsansätze wird an dieser Stelle aus Platzgründen verzichtet. Ein kritischer Überblick findet sich u.a. bei Fix, Opiłowski, Jakobs, Rolek.

${ }^{2}$ Zur Kritik des Konzeptes von R. de Beaugrande/W. Dressler siehe u. a. Linke/Nussbaumer (111), Heinemann (25).

${ }^{3}$ Allerdings gebührt K. Zimmermann das Verdienst, Intertextualität in die Sprachwissenschaft eingeführt zu haben. In seinem 1978 publizierten Aufsatz zur Texttypologie nahm er sich - obwohl eher fragmentarisch - der „Interaktion der Texte“ (Zimmermann 186) an, die er Intertextualität nannte.

${ }^{4}$ Ausführlicher dazu Nycz (,Vernetzung“ 401ff.)
} 
„Systematisierung potentieller Intertextualitätsindikatoren“ (34) anstrebt und ein „rezeptionsorientiertes Konzept von Intertextualität“ (6) vorstellen will, das - im Unterschied zu anderen Ansätzen - die Rolle des Rezipienten, seine intertextuelle Kompetenz bei der Erfassung und Verarbeitung intertextueller Beziehungen deutlich hervorhebt. Sie fasst Intertextualität als eine Texten

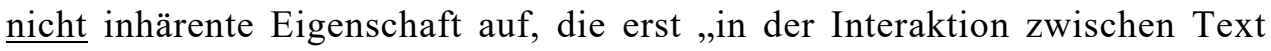
und Leser“, „im Kontinuum der Rezeption und nicht [...] im und durch den Text selbst" (31) konstituiert wird. Zugleich sind im Text bestimme Intertextualitätssignale vorhanden, die sog. ,intertextuelle Disposition des Textes“ (32), die ,abhängig von entsprechenden Interpretationshypothesen und -zielsetzungen des Rezipienten sowie spezifischen intertextuellen Wissensbeständen, aktiviert werden können“ (32). ${ }^{5}$

Weitere Versuche, unterschiedliche Ausprägungen intertextueller Beziehungen zu beschreiben und zu klassifizieren, wurden nach Holthuis mehrfach unternommen (z. B. bei Raible, Rößler, Jakobs, Fix). Doch in aller Regel werden die bereits bekannten Relationen lediglich mit neuen Begriffen versehen, sodass die von S. Holthuis vorgeschlagene Taxonomie m. E. immer noch als eines der umfassendsten und detailliertesten Konzepte gilt.

\section{VERNETZUNG VON (FACH-)TEXTSORTEN}

Während es sich im Falle der Intertextualität bekanntlich um Beziehungen zwischen Einzeltexten bzw. zwischen Text und Textmuster bzw. Textsorte handelt, bezieht sich das in der Überschrift angesprochene Phänomen auf Beziehungen zwischen Textsorten. Auf das Desiderat in der Erforschung dieser Relationen hat K. Adamzik (Textsorten 109) aufmerksam gemacht:

Die m. E. wesentlichste Beschreibungskategorie, um die die Textsortenforschung dringend erweitert werden sollte, betrifft das Kriterium der Einbettung von Textsorten in umfassendere kommunikative Strukturen und ihre Vernetztheit miteinander. Denn Textsorten bilden [...] strukturierte Subsysteme und gehören zu bestimmten Interaktions- oder Diskursrahmen. Zur Bewältigung einer kommunikativen Aufgabe können verschiedene Textsorten benutzt werden (diese stehen also in paradigmatischer Relation wie z.B. eine Werbeanzeige, ein Werbeplakat oder ein Werbebrief); häufig muss man auch eine ganze Reihe von Textsorten nacheinander bzw. grob gesprochen ,gleichzeitig“ produzieren, um eine komplexe kommunikative Aufgabe zu erfüllen.

\footnotetext{
${ }^{5}$ Zur Kritik der von S. Holthuis entwickelten Taxonomie siehe u.a. Tegtmeyer, Heinemann.
} 
Erste - teils theoretische, teils empirische - Arbeiten, die sich der Entstehung von Textsortennetzen annehmen, sind bereits in den 1990er Jahren entstanden. J. M. Swales erörterte die Vernetztheit von Textsorten am Beispiel des wissenschaftlichen Zeitschriftenaufsatzes, J. Klein („Politische Textsorten”, „Intertextualität") entwickelte ein Textsortennetz für den Bereich der Gesetzgebung und um die Folge einer TV-Soap-Opera. Auf „die systematischen Beziehungen zwischen Textsorten und Textsorten“ machte ebenfalls N. Janich („Intertextualität“ 193) aufmerksam. Für deren Beschreibung verwendete sie zunächst den von J. Klein („Intertextualität“) geprägten Terminus der Textsorten-Intertextualität. Sie verwies in diesem Zusammenhang darauf, dass die Vernetzung von Textsorten über „konkrete inhaltliche oder formale Bezugnahmen“ (Janich, „Intertextualität“ 194) hinausgehe. Es handele sich dabei vielmehr um ,syntagmatische und paradigmatische Beziehungen, die [...] verfahrensbedingt festgelegt sein können und damit unter Umständen sogar als textsortenkonstitutiv betrachtet werden müssen“ (194). Für die Beschreibung der Textsorten-Intertextualität schlug sie unter direktem Bezug auf K.-D. Baumann und H. Kalverkämper alternativ den Terminus Textsorten-in-Vernetzung vor. Zugleich stellte sie ein umfassendes Modell zur systematischen Beschreibung vernetzter Textsorten dar und erläuterte dieses (in einem späteren Aufsatz) an einer Fallstudie aus dem Bereich der Unternehmenskommunikation (vgl. Janich, „Zur Analyse“).

Die Erforschung der Vernetzung von Fachtexten bzw. Fachtextsorten, wie sie sich gegenwärtig als einer der aktuellsten Schwerpunkte in der Fachkommunikationsforschung etabliert, hat in den letzten Jahren zahlreiche wissenschaftliche Impulse erhalten. Bereits in den 2000er Jahren lieferte K.-D. Baumann (,Fachtexte-in-Vernetzung“, „Fachliche Intertextualität“) die theoretische Fundierung des Fachtexte-in-Vernetzung-Ansatzes. Die fachliche Intertextualität ist nach seiner Auffassung als „eine grundlegende Konstituente des Kommunikationsprozesses“ (Baumann, „Fachliche Intertextualität“ 143) zu verstehen, bei dem es „um den optimalen Transfer fachbezogener Informationen zwischen Fachleuten und Fachleuten [...] bzw. Fachleuten und Nichtfachleuten [...] geht" (143). K.-D. Baumann bietet ein dynamisches prozessuales Verständnis fachlicher Intertextualität, die eine intensive Interaktion von Kommunikationspartnern voraussetzt und das Resultat vieler ineinandergreifender kommunikativ-kognitiver Prozesse darstellt (144). Die systematische Beschreibung fachlicher Intertextualität umfasst Analysen strukturell-funktionaler und kommunikativ-kognitiver Bezugsmechanismen im Bereich einzelner Fachtexte, zwischen Fachtexten einer Fachtextsorte sowie zwischen Fachtexten mehrerer Fachtextsorten, wobei die Fachtextsortenvernetzung erst im Falle der zuletzt genannten Variante manifest wird (vgl. 144). 
Metalinguistische Kenntnisse der Textproduzenten und -rezipienten über prototypische „Relationsmuster bzw. Vernetzungsregularitäten von Fachtext(sort)en“, über „die präzise Produktion und Rezeption von Zitaten, Inhaltsangaben, Zusammenfassungen“ (145) spielen nach Baumanns Auffassung „eine kommunikationspraktische Schlüsselrolle“ (145). Da fachliche Intertextualität „das Ergebnis eines außerordentlich komplexen Zusammenspiels unterschiedlicher Bereiche der Fachkommunikation“ (160) darstellt und deren Erforschung Berücksichtigung einer Vielzahl von Beschreibungsdimensionen voraussetzt, entwickelte K.-D. Baumann ein äußerst komplexes interdisziplinäres Modell fachlicher Intertextualität mit 14 Bezugsebenen (vgl. 146ff.).

In späterer Zeit sind vereinzelte Aufsätze entstanden (z. B. Schippel, Nycz, „Vernetzung"), die die Vernetzung von Fachtext(sort)en zum Gegenstand hatten. Eine umfassendere über einen Aufsatz hinausgehende Untersuchung bot V. Ostapenko, in der sie sich mit der Vernetzung von Fachtextsorten der Normung in der technischen Harmonisierung auseinandersetzte. Den aktuellsten Schritt auf dem Weg zu einer komplexen Erfassung des Phänomens der Fachtextsorten-inVernetzung stellt der 2020 von H. Kalverkämper und K.-D. Baumann herausgebrachte Sammelband dar. In dem Band wird eine umfassende begrifflich-theoretische Explikation des Ansatzes Fachtextsorten-in-Vernetzung geliefert sowie eine methodologische Grundsatzdiskussion geführt, in der die relevanten Dimensionen der Fachtextsortenvernetzung, einschließlich der Dimension der (Fach-)Kultur systematisch erfasst werden. Die Autorinnen und Autoren des Bandes zeigen zudem an exemplarischen Beispielen aus der Fachkommunikation (Bibel und theologische Texte, juristische, medizinische Texte), wie sich unterschiedliche Textsorten innerhalb spezifischer Fächer miteinander vernetzen.

\section{VERNETZUNG VON FACHTEXTSORTEN IN DER BÖRSENKOMMUNIKATION}

Wegen der Komplexität des Kommunikationsbereiches Wirtschaft ${ }^{6}$ möchte ich diesen im vorliegenden Aufsatz auf die Börsenkommunikation und deren zentrale Textsorten eingrenzen.

Bei Börsenkommunikation ist von folgenden Kommunikationsräumen auszugehen:

\footnotetext{
${ }^{6}$ Ausführlicher dazu Nycz (Informationsstrukturierung 98f.)
} 
1. InStitutioneller KommunikationSRAum: umfasst sämtliche Kommunikationsformen zwischen börsennotierten Unternehmen und Wirtschafts-, Finanz- und Börsenmedien, privaten und institutionellen Anlegern bzw. Anteilseignern, Finanzinstituten, Börsenmaklern, Analysten, Rating-Agenturen u.a. zwecks Übermittlung markt- und unternehmensrelevanter Informationen. Wesentliche Teile der Kommunikation auf dieser Ebene werden durch Gesetze und Verordnungen, u.a. durch das Aktien-, Wertpapierhandels- und Börsengesetz, geregelt.

2. Nichtinstitutioneller KommunikATIONSRAUM, darunter

a. OfFizieller KommuniKationsRaum: Dazu gehört die Vermittlung von börsenrelevanten Informationen in Börsen-, Marktberichten, Analysen, Kommentaren v.a. mittels meinungsbildender Finanz- Wirtschafts- und Börsenmedien (Finanz- und Wirtschaftspresse, elektronische Informationsverbreitungssysteme, unabhängige Anbieter von Wirtschaftsnachrichten, z.B. dpa-afx.de);

b. INOFFIZIELLER KommunikATIONSRAUM: Diese Ebene der Börsenkommunikation betrifft den Austausch von größtenteils persönlichen Meinungen, Erfahrungen, Vermutungen (auch mit Elementen werblicher Aussagen) unter allen am Börsenhandel Interessierten (Wertpapierhändler, Anleger, Investoren usw.) auf Diskussionsforen, in Blogs, Communities/ Newsgroups im Internet u.a. (vgl. Nycz, Informationsstrukturierung 100).

$\mathrm{Zu}$ den zentralen Textsorten im Bereich der nichtinstitutionellen offiziellen Börsenkommunikation gehören - wie bereits angedeutet - Börsen- und Marktberichte sowie Wirtschaftskommentare. Während Börsenberichte über Verlauf und allgemeine Tendenz im Handel mit Wertpapieren sachlich und neutral informieren, gehen Marktberichte über den Effektenhandel hinaus und berichten zusätzlich über die Wirtschaftslage auf anderen Märkten. Beide Textsorten stützen sich auf amtliche Preisnotierungen. In Bezug auf die funktionale Textsortentypologie von Rolf (184) sind Berichte im Allgemeinen in den Bereich der darstellenden und registrierenden Textsorten einzuordnen. Nach Lüger (74f.) steht im Mittelpunkt eines Berichts ein bestimmter Sachverhalt, der als „chronologisch geordnete Folge von Handlungssequenzen“ (74) dargestellt wird, wobei diese chronologische Abfolge durch Zitate, kommentierende Stellungnahmen bzw. Hintergrundinformationen unterbrochen werden kann. Demgegenüber dienen Kommentare der Meinungsäußerung des Textautors, enthalten - der Zuordnung zu den judizierenden Textsorten im Sinne Rolfs entsprechend - eine kritische Stellungnahme bzw. Einschätzung eines Fachmanns zum aktuellen Börsengeschehen und sollten 
grundsätzlich das Denken und Handeln der Leser beeinflussen und sie dazu bringen, sich ihre eigene Meinung zum behandelten Sachverhalt zu bilden.?

Die bereits angesprochenen Zitate und kommentierenden Stellungnahmen der Aktienhändler in Börsen- und Marktberichten stellen die klassischen, explizit nachweisbaren Formen der Text-Text-Beziehung (ausführlich zum Zitat vgl. Holthuis 92ff.) dar. Ihr zentraler Zweck ist es, Händlermeinungen und Einschätzungen über das gegenwärtige und zukünftige Börsengeschehen wiederzugeben, über die wirtschafts- und politikrelevanten Einflussgrößen, die sich auf die Stimmung auf dem Markt auswirken zu reflektieren. Fremde Aussagen werden hierbei als Referenzen in praesentia (vgl. 93) in folgenden Formen signalisiert:

a) in Form von wörtlichen Zitaten:

Beispiel 1: DAX-FLASH: Geopolitik und Corona-Welle verunsichern Anleger (17.06.2020, www.dpa-afx.de, Abruf am 17.06.2020)

[...] Nach dem Corona-Ausbruch auf einem Großmarkt in Peking wurde die chinesische Hauptstadt inzwischen teilweise abgeriegelt. „China war als erstes Land von Corona betroffen, jetzt hat China als erstes Land die zweite Welle", sagte Marktexperte Thomas Altmann von QC Partners. „Die Frage ist jetzt, ob diese zweite Welle auch vielen anderen Ländern bevorsteht." Erneute wirtschaftliche Abriegelungen würden die ohnehin trüben Wirtschaftsausblicke noch mehr eintrüben, so Altmann. [...] (Hervorhebung - K.N.)

b) modifiziert als indirekte Rede mit dem Konjunktiv I:

Beispiel 2: Aktien Frankfurt Ausblick: Geopolitische Spannungen und Corona verunsichern (17.06.2020, www.dpa-afx.de, Abruf am 17.06.2020)

[...] Nach dem Corona-Ausbruch auf einem Großmarkt in Peking wurde die chinesische Hauptstadt zudem inzwischen teilweise abgeriegelt. China sei als erstes Land von Corona betroffen gewesen, jetzt drohe China als erstes Land die zweite Welle, sagte Marktexperte Thomas Altmann von QC Partners. Die Frage sei nun, ob diese zweite Welle auch anderen Ländern bevorsteht. Erneute Abriegelungen würden die ohnehin trüben konjunkturellen Aussichten noch mehr eintrüben. [...] (Hervorhebung - K.N.)

c) unmarkiert mit indikativischen Formen (meist unter Angabe des Autors bzw. der Autoren):

Beispiel 3: ROUNDUP/Aktien Frankfurt Eröffnung: Anleger bleiben trotz Corona zuversichtlich (17.06.2020, www.dpa-afx.de, Abruf am 17.06.2020)

\footnotetext{
${ }^{7}$ Ausführlicher zum Kommentar vgl. Lüger, zum Börsenbericht und -kommentar vgl. Nycz (Fachterminologie, Informationsstrukturierung).
} 
[...] Nach Einschätzung der Charttechnik-Experten der schweizerischen Bank UBS sind die Bullen, also die Optimisten, damit wieder stärker geworden. Mit dem Sprung über die runde 12 000-Punkte-Marke hätten sie ein sehr gutes Polster geschaffen. [...] (Hervorhebung - K.N.)

Wie den angeführten Textbeispielen zu entnehmen ist, werden gelegentlich unterschiedliche Ausdrucksmöglichkeiten der Redewiedergabe miteinander kombiniert. Es lassen sich keine plausiblen Hypothesen darüber aufstellen, nach welchen Kriterien die Textautoren bei der Wahl des Zitats, des Konjunktiv I bzw. des Indikativs vorgehen. Der Verwendung der einen oder anderen Möglichkeit der Redewidergabe liegen wohl teils stilistische, teils funktionale Aspekte zugrunde.

Die zuvor explizierten Markt- und Börsenberichte sowie Kommentare bilden im Bereich der nichtinstitutionellen, offiziellen Börsenkommunikation eine paradigmatisch-komplementäre Relation. Selbstverständlich sind sie in einem bestimmten Handlungsrahmen nicht austauschbar, verfolgen aber ein ähnliches Globalziel (,über einen Gegenstandsbereich informieren“), weisen sowohl thematisch als auch funktional Ähnlichkeiten auf und werden parallel zueinander produziert (vgl. auch Adamzik, „Textsortennetze“ 372ff., Klein, „Intertextualität“ 36).

In den nichtinstitutionellen, offiziellen Kommunikationsraum sind ferner weitere börsenrelevante Textsorten (etwa Analysten-Berichte und Peer Reviews) einzuordnen, die zu den vorstehend erwähnten Markt-, Börsenberichten und Kommentaren ebenfalls im paradigmatisch-komplementären Verhältnis stehen.
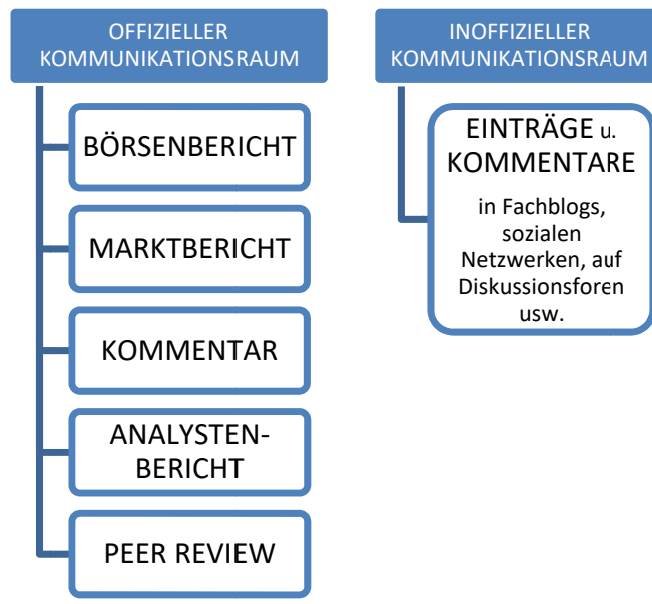

Abb. 1 
Die aufgeführten Textsorten stehen allesamt im engen syntagmatischen Beziehungsgefüge zu Kurstabellen, da die Tabellenwerte in den fraglichen Berichten und Kommentaren in expliziter Form interpretiert werden.

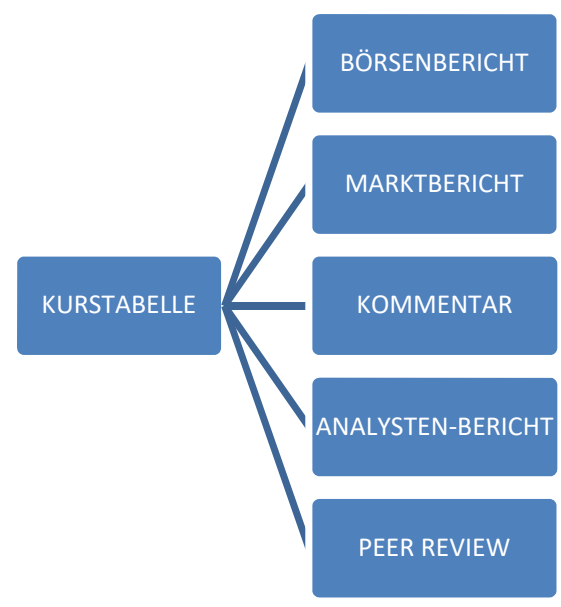

Abb. 2

In Kurstabellen, die börsentäglich in den Wirtschaftsteilen der Tagespresse und anderen (Online-)Medien veröffentlicht werden, werden die Entwicklungen der wichtigsten Aktienindizes und der am meisten gehandelten Aktien in komprimierter Form dargestellt. Bei Kurstabellen handelt es sich um eine besonders börsenrelevante Textsorte, die sich durch höchste Informationsdichte, Ausdrucksökonomie und zugleich größte Exaktheit auszeichnet. Allerdings sind Kurstabellen nur dann ein hilfreiches Werkzeug, wenn man die einzelnen Einträge kognitiv verarbeiten d. h. sinnvolle $\mathrm{Zu}$ sammenhänge zwischen Namen, Werten und Abkürzungen herstellen kann. ${ }^{8}$

Kurstabellen gelten einerseits als Bezugstexte für andere Texte meist im Bereich der nichtinstitutionellen, offiziellen Börsenkommunikation (s.o. Börsenberichte, Marktberichte, Kommentare), andererseits stehen sie als Posttexte bzw. Phänotexte (vgl. Janich, „Intertextualität“ 178) in syntagmatischer Relation zu Texten einer anderen Textsorte, zu dem sog. amtlichen Kursblatt.

\section{AMTLICHES KURSBLATT}

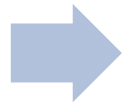

KURSTABELLE

Abb. 3

\footnotetext{
${ }^{8}$ Zur Kurstabelle als Textsorte siehe Hundt (649).
} 
Da das amtliche Kursblatt als ein offizielles Pflichtorgan gilt, das jede deutsche Börse regelmäßig, meist an jedem Börsentag herausgeben muss, ist es im Bereich der institutionellen Börsenkommunikation anzusiedeln. Das amtliche Kursblatt ist ein mehrere hundert Seiten umfassendes Dokument und bietet primär eine Übersicht über die verbindlichen fortlaufenden Kurse aller an der jeweiligen Börse notierten Wertpapiere. Darüber hinaus dient es der Veröffentlichung wichtiger Informationen der Börsenorgane sowie der Pflichtmitteilungen der Emittenten der Wertpapiere, etwa der Bekanntmachung der Hauptversammlungstermine (siehe z. B. Kursblatt). In Börsen- und Tageszeitungen (meist in deren Online-Ausgaben) werden Kursblätter im Auszug als Kurstabellen veröffentlicht.

Weitere Textsorten im Bereich der institutionellen Börsenkommunikation konstituieren ein Netzwerk, das sich durch paradigmatisch-komplementäre einerseits und syntagmatische Beziehungen andererseits auszeichnet. Eine zum Teil syntagmatische Relation besteht zwischen Quartals- und Geschäftsberichten. Quartalsberichte werden vierteljährlich veröffentlicht und enthalten eine Bilanz-, Gewinn- und Verlustrechnung, eine Kapitalflussrechnung sowie weitere Informationen über die Geschäftstätigkeit und Unternehmen. Der vierte Quartalsbericht wird in der Regel durch den Jahresabschluss ersetzt, der ein Bestandteil des Geschäftsberichtes darstellt (vgl. Quartalsberichterstattung). Geschäftsberichte sind komplexe Makrotexte, die diverse Rechnungsunterlagen umfassen. Zu den einzelnen Teilen des Geschäftsberichts finden sich im Aktiengesetz weitere Spezifizierungen (z. B. Lagebericht - §286 AktG, Jahresabschluss - § 149 AktG, Bericht des Aufsichtsrats - §171 Abs. 2 AktG, Gewinnverwendungsvorschlag - § 170 Abs. 2 AktG, Entsprechenserklärung - $§ 161 \mathrm{AktG})$. Ergänzend zur Berichterstattung des Unternehmens werden Aktionärsbriefe als Zwischenberichte veröffentlicht, in denen Aktiengesellschaften ihre Aktionäre über relevante Entwicklungen und den aktuellen sowie künftigen Geschäftsverlauf informieren (vgl. Aktionärsbrief). Eine Ergänzung zu den vorstehend explizierten Quartals- und Geschäftsberichten stellen ebenfalls Ad-hoc-Mitteilungen dar, in denen die Emittenten von Finanzinstrumenten alle Tatsachen unverzüglich bekanntzugeben haben, die sich auf die Wirtschaftslage des Unternehmens auswirken und folglich zu einer erheblichen Kursänderung der Aktien führen könnten. Als kursrelevante Tatsachen gelten sämtliche Insiderinformationen, in etwa Großaufträge, Directors' Dealings, Gewinneinbrüche, bedeutende personelle Veränderungen usw. Ad-hoc-Publizitätspflicht ist im Wertpapierhandelsgesetz ( $§ 15 \mathrm{WpHG})$ geregelt, das wiederum auf Art. 42 der Verordnung (EU) Nr. 600/2014 referiert (vgl. Ad-hoc-Publizität). 


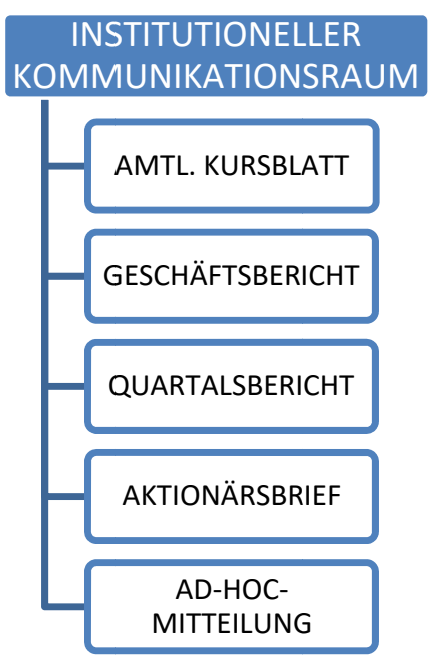

Abb. 4

Im Rahmen der nichtinstitutionellen, inoffiziellen Börsenkommunikation erfolgt der Austausch von Informationen bzw. eine Diskussion über aktuelle Kursnotierungen primär durch kurze Einträge und Kommentare in Fachblogs, sozialen Netzwerken und auf Diskussionsforen (syntagmatische Relation zu sämtlichen Textsorten aus dem institutionellen und nichtinstitutionellen offiziellen Kommunikationsraum). Diese Kommunikationsformen, die noch vor kurzem eher im privaten Bereich eine Rolle spielten und in der Fachkommunikation von geringer Relevanz waren, sind zu deren festem Bestandteil und Mittel geworden. Mittlerweile sind die meisten Unternehmen egal ob groß oder klein - auf Facebook, Twitter, Instagram oder LinkedIn präsent und versuchen mit unterschiedlichen Instrumenten Einfluss auf alle Interessenten zu nehmen.

\section{ABSCHLIESSENDE BEMERKUNG}

Die Vielfalt und zugleich Vielschichtigkeit intertextueller Beziehungen zwischen den vorstehend nur skizzenhaft dargestellten Fachtextsorten im Bereich der Börsenkommunikation weisen auf die Komplexität des Untersuchungsgegenstandes, gleichzeitig dessen enormes Erkenntnispotenzial hin. Eine solide, wissenschaftlich fundierte Untermauerung der Vernetzung von Fachtextsorten wurde bislang in einer Reihe von wissenschaftlichen Beiträ- 
gen (zu den neusten Erkenntnissen siehe H. Kalverkämper/K.-D. Baumann) geliefert. Weiteren Untersuchungen bleibt es nun vorbehalten, die Vernetztheit von Fachtextsorten in bestimmten Kommunikationsrahmen empirisch zu erforschen.

\section{LITERATUR}

Adamzik, Kirsten. „Textsortennetze.“ Textsorten, Handlungsmuster, Oberflächen. Linguistische Typologien der Kommunikation, hrsg. von Stephan Habscheid, De Gruyter Verlag, 2011, S. 367-385.

Adamzik, Kirsten. Textsorten. Reflexionen und Analysen. Stauffenburg Verlag, 2000.

„Ad-hoc-Publizität“. www.wirtschaftslexikon.co, Abruf am 18.06.2020.

„Aktionärsbrief“. www.wirtschaftslexikon.co, Abruf am 18.06.2020.

Baumann, Klaus-Dieter. „Fachtexte-in-Vernetzung aus interdisziplinärer Sicht.“ Fachtextsorten Gestern und Heute. Ingrid Wiese zum 65. Geburtstag, hrsg. von Irmhild Barz, Ulla Fix, Peter Lang Verlag, 2008, S. 109-127.

Baumann, Klaus-Dieter. „Fachliche Intertextualität - ein interdisziplinärer Untersuchungsansatz.“ Fach - Translat - Kultur. Interdisziplinäre Aspekte der vernetzten Vielfalt, hrsg. von KlausDieter Baumann, Frank \& Timme Verlag, 2011, S. 125-169.

„Börsenkommunikation“. https://wirtschaftslexikon.gabler.de, Abruf am 16.6.2020.

Brinker, Klaus. Textlinguistik. Groos Verlag, 1993.

De Beaugrande, Robert, u. Wolfgang Ulrich Dressler. Einführung in die Textlinguistik. Narr Verlag, 1981.

Fix, Ulla. „Aspekte der Intertextualität.“ Text- und Gesprächslinguistik, hrsg. von Klaus Brinker, Gerd Antos, Wolfgang Heinemann und Sven F. Sager, 1. Halbband, De Gruyter Verlag, 2000, S. 449-457.

Heinemann, Wolfgang. „Zur Eingrenzung des Intertextualitätsbegriffes.“ Textbeziehungen. Linguistische und literaturwissenschaftliche Beiträge zur Intertextualität, hrsg. von Josef Klein, Ulla Fix, Stauffenburg Verlag, 1997, S. 21-37.

Heinemann, Wolfgang, u. Dieter Viehweger. Textlinguistik. Eine Einführung. Niemeyer Verlag, 1991.

Holthuis, Susanne. Intertextualität. Aspekte einer rezeptionsorientierten Konzeption. Stauffenburg Verlag, 1993.

Hundt, Markus. „Textsorten des Bereichs Wirtschaft und Handel.“ Text- und Gesprächslinguistik, hrsg. von Klaus Brinker, Gerd Antos, Wolfgang Heinemann und Sven F. Sager, 1. Halbband, De Gruyter Verlag, 2000, S. 642-658.

Jakobs, Eva-Maria. Textvernetzung in den Wissenschaften. Niemeyer Verlag, 1999.

Janich, Nina. „Intertextualität und Text(sorten)vernetzung.“ Textlinguistik. 15 Einführungen, hrsg. von Nina Janich, Narr Verlag, 2008, S. 177-196.

Janich, Nina. „Zur Analyse von Textsorten-in-Vernetzung. Eine Modelldiskussion an einem Fallbeispiel aus der Unternehmenskommunikation." LAUD-Paper 734 (Series A: General and Theoretical Papers), 2009. 
Kalverkämper, Hartwig, u. Klaus-Dieter Baumann, Herausgeber. Fachtextsorten-in-Vernetzung. Frank \& Timme Verlag, 2020.

Klein, Josef. „Politische Textsorten.“ Aspekte der Textlinguistik, hrsg. von Klaus Brinker, Olms Verlag, 1991, S. 245-278.

Klein, Josef. „Intertextualität, Geltungsmodus, Texthandlungsmuster. Drei vernachlässigte Kategorien der Textsortenforschung - exemplifiziert an politischen und medialen Textsorten." Textsorten. Reflexionen und Analysen, hrsg. von Kirsten Adamzik, Stauffenburg Verlag, 2000, S. 31-44.

„Kursblatt“. www.boersenag.de, Abruf am 18.06.2020.

Linke, Angelika, u. Markus Nussbaumer. „Intertextualität. Linguistische Bemerkungen zu einem literaturwissenschaftlichen Textkonzept.“ Die Zukunft der Textlinguistik. Traditionen, Transformationen, Trends, hrsg. von Gerd Antos, Niemeyer Verlag, 1997, S. 109-126.

Lüger, Heinz-Helmut. Pressesprache. Niemeyer Verlag, 1983.

Nycz, Krzysztof. „Vernetzung von (Fach-)Texten.“ Fachkommunikation im Fokus. Paradigmen, Positionen, Perspektiven, hrsg. von Hartwig Kalverkämper, Frank \& Timme Verlag, 2016, S. 389-418.

Nycz, Krzysztof. Fachterminologie als Mittel des Fachwissenstransfers. Dr. Kovač Verlag, 2009

Nycz, Krzysztof. Informationsstrukturierung in Wirtschaftstexten. Peter Lang Verlag, 2017.

Opiłowski, Roman. Intertextualität in der Werbung der Printmedien. Eine Werbestrategie in linguistisch-semiotischer Forschungsperspektive. Peter Lang Verlag, 2006.

Ostapenko, Valentyna. Vernetzung von Fachtextsorten. Textsorten der Normung in der technischen Harmonisierung. Frank \& Timme Verlag, 2007.

„Quartalsberichterstattung“. www.wirtschaftslexikon.gabler.de, Abruf am 18.06.2020.

Raible, Wolfgang. „Arten des Kommentierens - Arten der Sinnbildung - Arten des Verstehens. Spielarten der generischen Intertextualität“. Text und Kommentar, hrsg. von Jan Assmann, Burkhard Gladigow, Fink Verlag, 1995, S. 51-73.

Rolek, Bogusława. „Stan badań nad intertekstualności w niemieckiej lingwistyce tekstu - próba bilansu". Lingwistyka tekstu w Polsce i w Niemczech, hrsg. von Zofia Bilut-Homplewicz, Waldemar Czachur und Marta Smykała, Atut, 2009, S. 233-248.

Rolf, Eckard. Die Funktionen der Gebrauchstextsorten. De Gruyter Verlag, 1993.

Rößler, Elke. Intertextualität und Rezeption. Linguistische Untersuchung zur Rolle von Text-TextKontakten im Textverstehen aktueller Zeitungstexte. Peter Lang Verlag, 1999.

Schippel, Larisa. „Europäische Textsortennetze.“ Übersetzungsqualität: Kritik, Kriterien, Bewertungshandeln, hrsg. von Larisa Schippel, Frank \& Timme Verlag, 2006, S. 43-54.

Swales, John M. Genre Analysis. English in Academic and Research Settings. CUP, 1990.

Tegtmeyer, Henning. „Der Begriff der Intertextualität und seine Fassungen. Eine Kritik der Intertextualitätskonzepte Julia Kristevas und Susanne Holthuis'." Textbeziehungen. Linguistische und literaturwissenschaftliche Beiträge zur Intertextualität, hrsg. von Josef Klein und Ulla Fix, Stauffenburg Verlag, 1997, S. 49-81.

Wilske, Ludwig, u. Wolf-Dieter Krause. „Intertextualität als allgemeine und spezielle Texteigenschaft“. Wissenschaftliche Zeitschrift, 31/1987, H. 5, S. 890-895.

Zimmermann, Klaus. Erkundungen zur Texttypologie: mit einem Ausblick auf die Nutzung einer Texttypologie für eine Corpustheorie. Narr Verlag, 1978. 


\section{POZA TEKSTEM. \\ ODNIESIENIA INTERTEKSTOWE W TEKSTACH BIZNESOWYCH}

Streszczenie

Pojęcie intertekstualności, wprowadzone do badań literackich przez Julię Kristevą w latach sześćdziesiątych XX wieku, wywołało niezwykle ożywioną debatę. W niniejszym artykule na wstępie zaprezentowano różne koncepcje intertekstualności. Następnie wyjaśniono koncepcję sieci tekstów (specjalistycznych). Na zakończenie podjęto próbę zilustrowania powiązań intertekstualnych na przykładzie wybranych typów tekstów specjalistycznych z dziedziny komunikacji giełdowej.

Słowa kluczowe: intertekstualność; sieci tekstów; komunikacja specjalistyczna; komunikacja giełdowa.

\section{BEYOND THE TEXT: \\ INTERTEXTUAL REFERENCES IN BUSINESS TEXTS}

\section{Su m m ary}

The concept of intertextuality, introduced into literary theory by Julia Kristeva in the 1960s, triggered an extensive debate in the realm of academic literature. First part of this essay focuses on different conceptions of intertextuality. It is followed by the explanation of the approach of genre networks. Final part attempts to illustrate the variety of intertextual references between selected types of texts in the field of stock exchange communication.

Keywords: intertextuality; genre networks; specialised communication; stock exchange communication. 